\title{
A New Method to Calculate the Appropriateness Measures of Label Expressions in Uncertainty Model
}

\author{
Yang Liu, Xingfang Zhang* \\ School of Mathematical Sciences, Liaocheng University, Liaocheng, China \\ Email: 15006378513@139.com, "zhangxingfang2005@126.com
}

Received 27 February 2016; accepted 12 March 2016; published 16 March 2016

Copyright (C) 2016 by authors and OALib.

This work is licensed under the Creative Commons Attribution International License (CC BY).

http://creativecommons.org/licenses/by/4.0/

(c) (i) Open Access

\section{Abstract}

The appropriateness measure of label expression is a basal concept in uncertainty modelling based on label semantics theory for dealing with vague concepts. In the paper, the concept of disjunctive normal forms is presented. It is proved that each label expression is semantic equivalent to a disjunctive normal form. Further, a new method of calculating the appropriateness measures of label expressions is provided.

\section{Keywords}

Epistemic Vagueness, Label Semantics, Random Sets, Appropriateness Measure, Disjunctive Normal Form

\section{Subject Areas: Mathematical Analysis}

\section{Introduction}

It is well known that any concept in classical mathematics is established on a crisp set (i.e., Cantor set). Suppose a concept $Q$ is defined by a non-empty set $D$, then we say the statement that $a$ is $Q$, is true (or its truth value is 1 ) if $a \in Q$; or else, it is false (or its truth value is 0). In other words, classical mathematics is established on classical logic or two-valued logic. However, for some propositions we cannot judge that they are true or false, such as the following propositions are not all classical propositions:

1) A coin tossed will be heads;

2) John will be in New York tomorrow;

3) John with 30 hairs is a bandicoot;

\footnotetext{
${ }^{*}$ Corresponding author.
} 
4) John is a bandicoot.

There are various nonclassical propositions in real life. Lukasiewicz is first extended classical logic to threevalued logic as early as 1920. In 1933, A.N. Kolmogoroff presented the probability theory for dealing with a type of uncertainty called randomness [1] (such as the above nonclassical propositions (1) and (2)). Following that, probabilistic logic for dealing with random proposition was proposed by Nilsson [2] based on probability theory in 1986. The theory of fuzzy set was initialized by Zadeh via membership function in 1965 [3]-[5] for fuzzy concepts (such as concept of bandicoot in the above propositions (3) and (4)). Following that, many types of many-valued logic and fuzzy logic were presented, respectively, such as Lukasiewicz fuzzy logic [6] product fuzzy logic, $L$ logic [7] [8], possibilistic logic [9], BL logic [10], and MTL logic [11].

Although multi-valued logics, fuzzy logic [12]-[19] and probabilistic logic are well developed in theory aspect, an actual interpretation of truth value of proposition is controversial. For example, Elkan and Watkins oppose fuzzy logics [20]-[22], and claim that fuzzy logics have some disadvantages, e.g., it does not hold the law of excluded middle (i.e., $v(\neg \theta \vee \theta) \equiv 1$ ) in classical logic, where $\theta$ denotes a proposition; $\neg \theta$ denotes its negation of proposition $\theta ; \vee$ denotes disjunction; and $v(\neg \theta \vee \theta)$ denotes the truth value of proposition $\neg \theta \vee \theta$. Recently, the author of paper also discussed this problem [23].

In fact, Zadeh's approach is the extension of a concept by a fuzzy set which has a graded characteristic or membership function with values between 0 and 1 . This allows for intermediate membership (values in $(0,1)$ ) in vague concepts resulting in intermediate truth values for propositions involving vague concepts (fuzzy logic). The calculus for fuzzy set theory is truth-functional which means that the full complement of Boolean laws cannot all be satisfied [24]. Furthermore, fuzzy set theory and fuzzy logic adopt an epistemic view of vagueness. Considering the shortcoming of fuzzy logic, it was proposed to the probabilistic logic holding the law of excluded middle dealing with fuzzy (or vague) concepts from a point of view in these papers [25]-[29]. In 2004, Lawry also provided a framework for linguistic modelling for dealing with vague (i.e. fuzzy) concepts based on label semantics using probability theory and random set [30]. At present it has been well developed [31]-[35] which was called uncertainty modelling for vague concepts in the paper [34]. In the theory, the appropriateness measure of label expressions is a basal concept. Given the label expression, a pivotal step of calculating the appropriateness measures is to seek a set of subsets of label corresponding to the label expression. Note that it is complicated to the approach of calculating the appropriateness measures of label expression provided in these papers [31]-[35]. Therefore the paper will discuss this problem.

The rest of this paper is organized as follows. Some basic concepts on uncertainty modelling for vague concepts are recalled in Section 2. In Section 3, the concept of disjunctive normal forms is first presented; then it is proved that each label expression is semantic equivalent to a disjunctive normal form; finally, a new method of calculating the appropriateness measure of label expression is provided. At the end of this paper, a brief summary is given.

\section{Preliminaries}

Definition 1 (Label expressions). Given a finite set of labels $L A$ the corresponding set of label expressions LE is defined recursively as follows:

- If $L \in L A$, then $L \in L E$;

- If $\theta, \varphi \in, L E$ then $\neg \theta, \theta \wedge \varphi, \theta \vee \varphi \in L E$.

The mass function $m_{x}$ on sets of labels then quantifies the agent's belief that any particular subset of labels contains all and only the labels with which it is appropriate to describe $x$ i.e. $m_{x}(F)$ is the agent's subjective probability that $D_{x}=F$.

Definition 2 (Mass function on labels). $\forall x \in \Omega$ a mass function on labels is a function $m_{x}: 2^{L A} \rightarrow[0,1]$ such that $\sum_{F \subseteq L A} m_{x}(F)=1$.

Definition 3 ( $\lambda$-mapping). $\lambda: L E \rightarrow 2^{2^{L A}}$ is defined recursively as follows: $\forall \theta, \varphi \in L E$

- $\forall L_{i} \in L A, \lambda\left(L_{i}\right)=\left\{F \subseteq L A \mid L_{i} \in F\right\}$.

- $\lambda(\theta \wedge \varphi)=\lambda(\theta) \cap \lambda(\varphi)$.

- $\lambda(\theta \vee \varphi)=\lambda(\theta) \cup \lambda(\varphi)$. 
- $\lambda(\neg \theta)=(\lambda(\theta))^{c}$.

Based on the $\lambda$ mapping we then define $\mu_{\theta}(x)$ as the sum of $m_{x}$ over those set of labels in $\lambda(\theta)$. The sum of $m_{x}$ over those set of labels in $\lambda(\theta)$.

Definition 4 (Appropriateness measure). The appropriateness measure defined by mass function $m_{x}$ is a function $\mu: L A \times \Omega \rightarrow[0,1]$ satisfying

$$
\forall \theta \in L E, \forall x \in \Omega, \mu_{\theta}(x)=\sum_{F \in \lambda(\theta)} m_{x}(F) .
$$

Let Val be the set of valuation functions $v: L A \rightarrow\{0,1\}$ where for $L_{i} \in L A, v\left(L_{i}\right)=1$ means that $L_{i}$ is appropriate in the current context. In particular, the epistemic stance dictates that for each $x \in \Omega$ there would be a corresponding valuation $v_{x}$ (partially unknown to the agent) determining which labels are appropriate to describe $x$. A valuation $v \in \mathrm{Val}$ naturally determines an extension $v: L A \rightarrow 0,1$ defined recursively as follows: For $\theta, \varphi \in L E ; v(\theta \wedge \varphi)=\min (v(\theta), v(\varphi)), v(\theta \vee \varphi)=\max (v(\theta))$, and $v(\neg \theta)=1-v(\theta)$. We can now define $\vDash$ and $\equiv$ as follows:

Definition 5. $\forall \theta, \varphi \in L E$

- $\theta \vDash \varphi$ if $\forall v \in \operatorname{Val}, v(\theta)=1$ then $v(\varphi)=1$.

- $\theta \equiv \varphi$ if $\forall v \in \operatorname{Val}, v(\theta)=v(\varphi)$.

- $\theta$ is a tautology, if $\forall v \in \operatorname{Val}, v(\theta)=1$.

- $\theta$ is a contradiction, if $\forall v \in \operatorname{Val}, v(\theta)=0$.

Theorem 6 (General properties of appropriateness measures). $\forall \theta, \varphi \in L E$ the following properties hold:

- If $\theta \vDash \varphi$ then $\forall x \in \Omega, \mu_{\theta}(x) \leq \mu_{\varphi}(x)$.

- $\theta \equiv \varphi$ then $\mu_{\theta}(x)=\mu_{\varphi}(x)$.

- If $\theta$ is a tautology, then $\mu_{\theta}(x)=1$.

- If $\theta$ is a contradiction, then $\mu_{\theta}(x)=0$.

- If $\theta \wedge \varphi$ is a contradiction, then $\mu_{\theta \vee \phi}(x)=\mu_{\theta}(x)+\mu_{\varphi}(x)$.

- $\forall \theta \in L A, \mu_{\neg \theta}(x)(x)=1-\mu_{\theta}(x)$.

- For $F \subseteq L A$, let $\theta_{F}=\left(\wedge_{L_{i} \in F} L_{i}\right) \wedge\left(\wedge_{L_{i} \in F} \neg L_{i}\right)$, then $\mu_{\theta}(x)=m_{x}(F)$.

We not find the proof of last property in Theorem 6 in these papers [30]-[35]. Therefore, now we provide it.

Proof. Without loss of generality, suppose $F=\left\{L_{1}, L_{2}, \cdots L_{j}\right\}, \theta_{F}=L_{1} \wedge L_{2} \wedge \cdots \wedge L_{j} \wedge \neg L_{j+1} \cdots \wedge \neg L_{n}$. Since it follows from Definition 3 that

$$
\lambda\left(\theta_{F}\right)=\lambda\left(L_{1}\right) \cap \lambda\left(L_{2}\right) \cap \cdots \cap \lambda\left(L_{j}\right) \cap \lambda\left(\neg L_{j+1}\right) \cap \lambda\left(\neg L_{j+2}\right) \cap \cdots \cap \lambda\left(\neg L_{n}\right) .
$$

Thus we only need to prove that

$$
\lambda\left(L_{1}\right) \cap \lambda\left(L_{2}\right) \cap \cdots \cap \lambda\left(L_{j}\right) \cap \lambda\left(\neg L_{j+1}\right) \cap \lambda\left(\neg L_{j+2}\right) \cap \cdots \cap \lambda\left(\neg L_{n}\right)=\{F\} .
$$

We first prove that

$$
\{F\} \subseteq \lambda\left(L_{1}\right) \cap \lambda\left(L_{2}\right) \cap \cdots \cap \lambda\left(L_{j}\right) \cap \lambda\left(L_{j+1}\right) \cap \lambda\left(L_{j+2}\right) \cap \cdots \cap \lambda\left(L_{n}\right) .
$$

Since for each $i \in\{1,2, \cdots, n\}, \lambda\left(L_{i}\right)=\left\{E \subseteq L A \mid L_{i} \in E\right\}, \lambda\left(\neg L_{i}\right)=\left(\lambda\left(L_{i}\right)\right)^{c}$, also, $\forall i \in\{1,2, \cdots, j\}$, $L_{i} \in F$ and $\forall i \in\{j+1, j+2, \cdots, n\}, \quad L_{i} \in F$ not holds, it follows that $F \in \lambda\left(L_{i}\right), \quad i=1,2, \cdots, j$ and $F \in \lambda\left(\neg L_{i}\right), \quad i=j+1, j+2, \cdots, n$. Therefore

$$
F \in \lambda\left(L_{1}\right) \cap \lambda\left(L_{2}\right) \cap \cdots \cap \lambda\left(L_{j}\right) \cap \lambda\left(L_{j+1}\right) \cap \lambda\left(L_{j+2}\right) \cap \cdots \cap \lambda\left(L_{n}\right) .
$$

Thus the formula (3) is true.

Now we prove that for any $E \in L A$, if $E \neq F$, then

$E \in \lambda\left(L_{1}\right) \cap \lambda\left(L_{2}\right) \cap \cdots \cap \lambda\left(L_{j}\right) \cap \lambda\left(L_{j+1}\right) \cap \lambda\left(L_{j+2}\right) \cap \cdots \cap \lambda\left(L_{n}\right)$ not holds. In fact, if $E$ not contain $L_{i}$, $i=1,2, \cdots, j$ then $E \in \lambda\left(L_{i}\right), i=1,2, \cdots, j$ not hold; if $E$ contain $L_{i}, i=j+1, j+2, \cdots, n$, then $E \in \lambda\left(\neg L_{i}\right)$, 
$i=j+1, j+2, \cdots, n$ not hold. In a word, $E \in \lambda\left(L_{1}\right) \cap \lambda\left(L_{2}\right) \cap \cdots \cap \lambda\left(L_{j}\right) \cap \lambda\left(L_{j+1}\right) \cap \lambda\left(L_{j+2}\right) \cap \cdots \cap \lambda\left(L_{n}\right)$ not holds.

Therefore $\lambda\left(\theta_{F}\right)=\{F\}$ is true. It follows that $\mu_{\theta}(x)=m_{x}(F)$.

The theorem is proved.

\section{Calculating of the Appropriateness Measures}

In the Section we first discuss the properties of valuation functions.

For convenience, we call each element in Label $L A=\left\{L_{1}, L_{2}, \cdots, L_{n}\right\}$ as atomic label expression. Let $\theta$ be a label expression containing atomic label expressions $L_{1}, L_{2}, \cdots, L_{k}$, then we can be denoted by $\theta\left(L_{1}, L_{2}, \cdots, L_{k}\right)$. Although it not contains atomic label expressions $L_{k+1}, L_{2}, \cdots, L_{n}$, we also can write it as $\theta\left(L_{1}, L_{2}, \cdots, L_{n}\right)$. The mapping $v: L A \rightarrow\{0,1\}$ is denoted by $v_{L A}$, and write $v_{L A}=\left(v_{L A}\left(L_{1}\right), v_{L A}\left(L_{2}\right), \cdots, v_{L A}\left(L_{n}\right)\right)$. For example, if $L A=\left\{L_{1}, L_{2}, L_{3}\right\}$, and $v_{L A}\left(L_{1}\right)=1, v_{L A}\left(L_{2}\right)=0, v_{L A}\left(L_{3}\right)=1$, then $v_{L A}=(1,0,1)$ is regard as a vector in $\{0,1\}^{n}$.

Note that $v_{L A}^{-1}(1)$ is a subset of $L A$, and a relation of one to one from the set Val of all this mapping to $\{0,1\}^{n}$ is gained, and the valuation function $v(\theta), v \in$ Val of $\theta$ is a Boolean function $f:\{0,1\}^{n} \rightarrow\{0,1\}$. Such function $f$ is denoted by $f_{\theta}\left(\xi_{1}, \xi_{2}, \cdots, \xi_{n}\right),\left(\xi_{1}, \xi_{2}, \cdots, \xi_{n}\right) \in\{0,1\}^{n}$. Where $\xi_{i}$ can be considered a random variable, $\left(\xi_{1}, \xi_{2}, \cdots, \xi_{n}\right)$ a $n$-dimensional random variable, and $f\left(\xi_{1}, \xi_{2}, \cdots, \xi_{n}\right)$ a function of $n$ random variables.

Definition 7. A label expression $\theta$ is said to be a disjunctive normal form, if its form is

$$
\left(Q_{11} \wedge Q_{12} \wedge \cdots \wedge Q_{1 n}\right) \vee\left(Q_{21} \wedge Q_{22} \wedge \cdots \wedge Q_{2 n}\right) \vee \cdots \vee\left(Q_{m 1} \wedge Q_{m 2} \wedge \cdots \wedge Q_{m n}\right),
$$

where $Q_{i j}$ is $L_{j}$ or $\neg L_{j}$, for $i, j=1,2, \cdots, n$, and $\left(Q_{11} \wedge Q_{12} \wedge \cdots \wedge Q_{1 n}\right),\left(Q_{21} \wedge Q_{22} \wedge \cdots \wedge Q_{2 n}\right), \cdots,\left(Q_{m 1} \wedge Q_{m 2} \wedge \cdots \wedge Q_{m n}\right)$ are all different.

Let $G=\left\{\left(Q_{11} \wedge Q_{12} \wedge \cdots \wedge Q_{1 n}\right) \mid Q_{11} \in\left\{L_{1}, \neg L_{1}\right\}, Q_{12} \in\left\{L_{2}, \neg L_{2}\right\}, \cdots, Q_{1 n} \in\left\{L_{n}, \neg L_{n}\right\}\right\}$, each $w \in G$ is called a conjoint atomic label expression.

Lemma 8. For each $v=\left(\xi_{1}, \xi_{2}, \cdots, \xi_{n}\right) \in \mathrm{Val}$, then $v\left(w_{0}\right)=1$, where $w_{0}=\left(Q_{11} \wedge Q_{12} \wedge \cdots \wedge Q_{1 n}\right) \in G$ satisfy that if $\xi_{i}=1 ; \quad Q_{11}=\neg L_{i}$ if $\xi_{i}=0$, and for $\forall w \in G-\{w\}$ we have $v(w)=0$.

Proof. Let $v=\left(\xi_{1}, \xi_{2}, \cdots, \xi_{n}\right) \in$ Val.

On the one hand, $v\left(L_{i}\right)=1$ if $\xi_{i}=1$; it follows from $v\left(L_{i}\right)=0$ that $v\left(\neg L_{i}\right)=1$, if $\xi_{i}=0$. Thus

$$
v\left(w_{0}\right)=\min \left\{v\left(Q_{11}\right), v\left(Q_{12}\right), \cdots, v\left(Q_{1 n}\right)\right\}=1 .
$$

On the other hand, let $w \in G-\left\{w_{0}\right\}$, then there exists $L_{j} \in L A$ or $\neg L_{j}$ is contained in $w_{0}$ and it is not contained in $w$. Suppose $L_{j} \in L A$ is contained in $w_{0}$ and it is not contained in $w$. Thus $\neg L_{j}$ is contained in $w$ and $\xi_{j}=1$. Thus $v(w)=0$.

Lemma 9. Let label expression $\theta$ be a non-contradiction, and it contains atomic label expressions $L_{1}, L_{2}, \cdots, L_{n}$. Then it is semantically equivalent to a disjunctive normal form as follows:

i.e.,

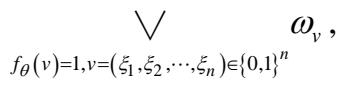

$$
\theta \equiv \underset{f_{\theta}(v)=1, v=\left(\xi_{1}, \xi_{2}, \cdots, \xi_{n}\right) \in\{0,1\}^{n}}{\vee} \omega_{v},
$$

where for each $v=\left(\xi_{1}, \xi_{2}, \cdots, \xi_{n}\right) \in\{0,1\}^{n}, f_{\theta}(v)=1$,

$$
\omega_{v}=Q_{1 v} \cap Q_{2 v} \cap \cdots \cap Q_{n v}
$$

satisfies that $Q_{i v}$ is $L_{i}$ if $\xi_{i}=1 ; Q_{i v}$ is $\neg L_{i}$ if $\xi_{i}=0$, for $i=1,2, \cdots, n$.

If

$$
\theta \equiv \underset{f_{\theta}(v)=1, v=\left(\xi_{1}, \xi_{2}, \cdots, \xi_{n}\right) \in\{0,1\}^{n}}{\bigvee} \omega_{v},
$$

we call

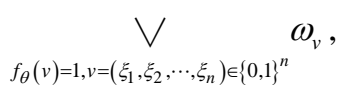


as disjunctive normal form of $\theta$, and it is denoted by $D(\theta)$.

Proof. From Definition 5 we need to prove

$$
f_{\theta}(v)=f_{D(\theta)}(v), v \in \text { Val. }
$$

It is evident that we only need to prove $f_{\theta}(v)=1$ iff $f_{D(\theta)}(v)=1, v \in$ Val.

For $\forall v=\left(\xi_{1}, \xi_{2}, \cdots, \xi_{n}\right) \in V a l$, suppose $f_{\theta}(v)=f_{\theta}\left(\xi_{1}, \xi_{2}, \cdots, \xi_{n}\right)=1$, then by Lemma 8 we have $v\left(Q_{1 v} \cap Q_{2 v} \cap \cdots \cap Q_{n v}\right)=1$. It follows from conjoint atomic label expression $\omega_{v}=\left(Q_{1 v} \cap Q_{2 v} \cap \cdots \cap Q_{n v}\right)$ is contained in

$$
\underset{f_{\theta}(v)=1,}{\bigvee} \omega_{v},
$$

that $v\left(\omega_{v}=Q_{1 v} \cap Q_{2 v} \cap \cdots \cap Q_{n v}\right)=1$, thus

$$
v\left(\underset{f_{\theta}(v)=1, v=\left(\xi_{1}, \xi_{2}, \cdots, \xi_{n}\right) \in\{0,1\}^{n}}{V} \omega_{\xi}\right)=1 .
$$

Contrarily, for $\forall v_{0}=\left(\xi_{1}, \xi_{2}, \cdots, \xi_{n}\right) \in V a l$, suppose

$$
v_{0}\left(\underset{f_{\theta}(v)=1, v=\left(\xi_{1}, \xi_{2}, \cdots, \xi_{n}\right) \in\{0,1\}^{n}}{\vee} \omega_{v}\right)=1 .
$$

By Lemma 8 we known that $v_{0}\left(Q_{1 v_{0}} \cap Q_{2 v_{0}} \cap \cdots \cap Q_{n v_{0}}\right)=1, \quad v\left(Q_{1 v} \cap Q_{2 v} \cap \cdots \cap Q_{n v}\right)=0$ if $v \in V a l-\left\{v_{0}\right\}$. Thus $Q_{1 v_{0}} \cap Q_{2 v_{0}} \cap \cdots \cap Q_{n v_{o}}$ is contain in

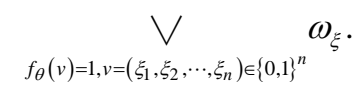

Thus $f_{\theta}\left(v_{0}\right)=1$.

The theorem is proved.

By Lemma 9 and Definition 3 we easily gained the following Lemma.

Lemma 10. Let $\forall x \in \Omega$ a mass function on labels $L A$ is a function $m_{x}: 2^{L A} \rightarrow[0,1]$ such that $\sum_{F \subseteq L A} m_{x}(F)=1$. Then

$$
\mu_{\theta}(x)=\sum_{f_{\theta}(v)=1, v=\left(\xi_{1}, \xi_{2}, \cdots, \xi_{n}\right) \in\{0,1\}^{n}} \mu_{\omega_{v}}(x),
$$

if

$$
D(\theta)=\bigcup_{f_{\theta}(v)=1, \xi=\left(\xi_{1}, \xi_{2}, \cdots, \xi_{n}\right) \in\{0,1\}^{n}} \omega_{v},
$$

where for each $v=\left(\xi_{1}, \xi_{2}, \cdots, \xi_{n}\right) \in\{0,1\}^{n}, f_{\theta}(v)=1$,

$$
\omega_{v}=Q_{1 \xi} \cap Q_{2 \xi} \cap \cdots \cap Q_{n \xi}
$$

satisfies that $Q_{i \xi}$ is $L_{i}$ if $\xi_{i}=1 ; Q_{i \xi}$ is $\neg L_{i}$ if $\xi_{i}=0$, for $i=1,2, \cdots, n$.

Theorem 11. Let $\forall x \in \Omega$ a mass function on labels $L A$ is a function $m_{x}: 2^{L A} \rightarrow[0,1]$ such that $\sum_{F \subseteq L A} m_{x}(F)=1$. For any $\theta \in L E$,

$$
\mu_{\theta}(x)=\sum_{f_{\theta}(\xi)=1, \xi=\left(\xi_{1}, \xi_{2}, \cdots, \xi_{n}\right) \in\{0,1\}^{n}} m_{x}\left(v_{L A}^{-1}(1)\right) .
$$

Proof. By Lemma 10 we have

$$
\mu_{\theta}(x)=\sum_{f_{\theta}(v)=1, v=\left(\xi_{1}, \xi_{2}, \cdots, \xi_{n}\right) \in\{0,1\}^{n}} \mu_{\omega_{v}}(x) .
$$

It follows from Theorem 6 and the meaning of mapping $v_{L A}$, foe each $v=\left(\xi_{1}, \xi_{2}, \cdots, \xi_{n}\right) \in\{0,1\}^{n}$, $f_{\theta}(v)=1, \mu_{\omega_{v}}(x)=m_{x}\left(v_{L A}^{-1}(1)\right)$, thus the theorem is true.

Exempla 12. Suppose $L A=\left\{L_{1}, L_{2}, L_{3}\right\}, x \in \Omega$, and $m_{x}: 2^{L A} \rightarrow[0,1]$ is a mass function on labels $L A$ satisfying: 


$$
\begin{aligned}
& m_{x}\left(\left\{L_{1}\right\}\right)=m_{x}\left(\left\{L_{3}\right\}\right)=\frac{1}{10} \\
& m_{x}\left(\left\{L_{2}\right\}\right)=\frac{2}{5} \\
& m_{x}\left(\left\{L_{1}, L_{2}\right\}\right)=m_{x}\left(\left\{L_{2}, L_{3}\right\}\right)=\frac{1}{5} \\
& =m_{x}\left(\left\{L_{1}, L_{3}\right\}\right)=m_{x}\left(\left\{L_{2}, L_{2}, L_{3}\right\}\right)=0 .
\end{aligned}
$$

For $\theta=\neg\left(L_{1} \vee \neg L_{2}\right) \vee L_{3}$, note that

$$
\left\{v=\left(\xi_{1}, \xi_{2}, \xi_{3}\right) \mid f\left(\xi_{1}, \xi_{2}, \xi_{3}\right)=1\right\}=\{(0,1,1),(0,1,0),(0,0,1),(1,1,1),(1,0,1)\} .
$$

It we write $v_{1}=(0,1,1), v_{2}=(0,1,0), v_{3}=(0,0,1), v_{4}=(1,1,1), v_{5}=(1,0,1)$, then

$$
\begin{aligned}
& v_{1}^{-1}(1)=\left\{L_{2}, L_{3}\right\} \\
& v_{2}^{-1}(1)=\left\{L_{2}\right\} \\
& v_{3}^{-1}(1)=\left\{L_{3}\right\} \\
& v_{4}^{-1}(1)=\left\{L_{1}, L_{2}, L_{3}\right\} \\
& v_{5}^{-1}(1)=\left\{L_{1}, L_{3}\right\} .
\end{aligned}
$$

Thus we have

$$
\begin{aligned}
& \mu_{\neg\left(L_{1} \vee \neg L_{2}\right) \vee L_{3}}(x) \\
& =m_{x}\left(\left\{L_{2}, L_{3}\right\}\right)+m_{x}\left(\left\{L_{2}\right\}\right)+m_{x}\left(\left\{L_{3}\right\}\right)+m_{x}\left(\left\{L_{1}, L_{3}\right\}\right)+m_{x}\left(\left\{L_{1}, L_{2}, L_{3}\right\}\right) \\
& =\frac{1}{5}+\frac{2}{5}+\frac{1}{10}=\frac{7}{10} .
\end{aligned}
$$

\section{Conclusion}

The paper manly provided a new method for calculating the appropriateness measures of label expressions. Based on the fact, each label expression is semantic equivalent to a disjunctive normal form.

\section{Acknowledgements}

This work was supported by national natural science foundation of China grant No.11471152 and No.61273044.

\section{References}

[1] Chen, L., Mu, Z.C. and Nan, B.F. (2015) Semantic Image Segmentation Based on Hierarchical Conditional Random Field Model. Journal of Computational Information Systems, 11, 527-534.

[2] Nilsson, N. (1986) Probability Logic. Artificial Intelligence, 28, 71-78. http://dx.doi.org/10.1016/0004-3702(86)90031-7

[3] Zadeh, L.A. (1973) Outline of a New Approach to the Analysis of Complex Systems and Decision Processes. IEEE Transactions on Systems, Man and Cybernetics, 3, 28. http://dx.doi.org/10.1109/TSMC.1973.5408575

[4] Zadeh, L.A. (1965) Fuzzy Sets. Information and Control, 8, 338-353. http://dx.doi.org/10.1016/S0019-9958(65)90241-X

[5] Zadeh, L.A. (1975) Fuzzy Logic and Approximate Reasoning. Synthese, 30, 407-428. http://dx.doi.org/10.1007/BF00485052

[6] Lukasiewicz, J. and Tarski, A. (1930) Untersuchungen über den Aussagenkalkül. Comptes Rendus des Séances de la Sociélé des Scierices et des Lettres des Varsovie Classe III, 23, 30-50.

[7] Wang, G. and Zhou, H. (2009) Introduction to Mathematical Logic and Resolution Principle. 2nd Edition, Science Press Beijing, and Alpha Science International Limited, Oxford.

[8] Wang, G. (1997) A Formal Deduction System of Fuzzy Propositional Calculation. Science in China Series E-Information Sciences, 42, 1041-1044. 
[9] Doubois, D. and Prade, H. (2001) Possibility Theory, Probability Theory and Multiple-Valued Logic. Annals of Mathematics and Artificial Intelligence, 32, 35-66. http://dx.doi.org/10.1023/A:1016740830286

[10] Hajek, P. (1998) Metamathematics of Fuzzy Logic. Kluwer Academic Publishers, London, 89-120. http://dx.doi.org/10.1007/978-94-011-5300-3

[11] Esteva, F. and Godo, L. (2001) Monoidal t-Norm Based Logic: Towards Logic for Left-Continuous t-Norms. Fuzzy Sets and Systems, 124, 271-288. http://dx.doi.org/10.1016/S0165-0114(01)00098-7

[12] Dubois, D. and Prade, H. (1988) An Introduction to Possibility and Fuzzy Logics. In: Smets, P., Mamdani, A., Dubois, D. and Prade, H., Eds., Non-Standard Logics for Automated Reasoning, Academic Press, London, 742-755.

[13] Dubois, D. and Prade, H. (1990) Measuring Properties of Fuzzy Sets: A General Technique and Its Use in Fuzzy Query Evaluation. Fuzzy Sets and Systems, 38, 137-152. http://dx.doi.org/10.1016/0165-0114(90)90146-W

[14] Dubois, D. and Prade, H. (1997) The Three Semantics of Fuzzy Sets. Fuzzy Sets and Systems, 90, 141-150. http://dx.doi.org/10.1016/S0165-0114(97)00080-8

[15] Dubois, D., Godo, L., Prade, H. and Esteva, F. (2005) An Information-Based Discussion of Vagueness. In: Cohen, H. and Lefebre, C., Eds., Handbook of Categorization in Cognitive Science, Elsevier Science, Amsterdam, 891-909. http://dx.doi.org/10.1016/B978-008044612-7/50095-0

[16] Li, L. and Zhang, J. (2010) Attribute Reduction in Fuzzy Concept Lattices Based on the T Implication Original Research Article Pages. Knowledge-Based Systems, 23, 497-503. http://dx.doi.org/10.1016/j.knosys.2010.03.006

[17] Miller, S. and John, R. (2010) An Interval Type-2 Fuzzy Multiple Echelon Supply Chain Model Original Research Article Pages. Knowledge-Based Systems, 23, 363-368. http://dx.doi.org/10.1016/j.knosys.2009.11.016

[18] Buckley, J., Siler, W. and Tucker, D. (1986) A Fuzzy Expert System. Fuzzy Sets and Systems, 20, 1-16. http://dx.doi.org/10.1016/S0165-0114(86)80027-6

[19] Egemen, A. and Telatar, Y.Z. (2010) Note-against-Note Two-Voice Counterpoint by Means of Fuzzy Logic Original Research Article. Knowledge-Based Systems, 23, 256-266. http://dx.doi.org/10.1016/j.knosys.2010.01.007

[20] Elkan, C. (1994) The Paradoxical Success of Fuzzy Logic. IEEE Expert, 9, 3-8. http://dx.doi.org/10.1109/64.336150

[21] Elkan, C. (1994) The Paradoxical Controversy over Fuzzy Logic. IEEE Expert, 9, 47-49. http://dx.doi.org/10.1109/64.336150

[22] Watkins, F.A. (1995) False Controversy: Fuzzy and Non-Fuzzy Faux Pas. IEEE Expert, 10, 4-5.

[23] Zhang, X. (2011) Duality and Pseudo Duality of Dual Disjunctive Normal Forms. Knowledge-Based Systems, 24, 1033-1036. http://dx.doi.org/10.1016/j.knosys.2011.04.017

[24] Zadeh, L.A. (1975) The Concept of Linguistic Variable and Its Application to Approximate Reasoning, Part 2. Information Sciences, 8, 301-357. http://dx.doi.org/10.1016/0020-0255(75)90046-8

[25] Flaminio, T. and Godo, L. (2007) A Logic for Reasoning about the Probability of Fuzzy Events. Fuzzy Sets and Systems, 158, 625-638. http://dx.doi.org/10.1016/j.fss.2006.11.008

[26] Coletti, G. and Scozzafava, R. (2002) Probability Logic in a Coherent Setting. Kluwer Academic Publishers, London. http://dx.doi.org/10.1007/978-94-010-0474-9

[27] Fine, K. (1975) Vagueness, Truth and Logic. Synthese, 30, 265-300. http://dx.doi.org/10.1007/BF00485047

[28] Hailperin, T. (1996) Sentential Probability Logic. Associated University Presses, London.

[29] Flaminioa, T. and Godo, L. (2007) A Logic for Reasoning about the Probability of Fuzzy Events. Fuzzy Sets and Systems, 158, 625-638. http://dx.doi.org/10.1016/j.fss.2006.11.008

[30] Lawry, J. (2004) A Framework for Linguistic Modeling. Artificial Intelligence, 155, 1-39. http://dx.doi.org/10.1016/j.artint.2003.10.001

[31] Lawry, J. (2006) Modelling and Reasoning with Vague Concepts. Springer, Berlin.

[32] Lawry, J. (2008) Appropriateness Measures: An Uncertainty Model for Vague Concepts. Synthese, 161, 255-269. http://dx.doi.org/10.1007/s11229-007-9158-9

[33] Lawry, J. (2008) An Overview of Computing with Words Using Label Semantics. In: Bustince, H., Herrera, F. and Montero, J., Eds., Fuzzy Sets and Their Extensions: Representation, Aggregation and Models, Springer, Berlin, 65-87. http://dx.doi.org/10.1007/978-3-540-73723-0_4

[34] Lawry, J. and Tang, Y. (2009) Uncertainty Modelling for Vague Concepts: A Prototype Theory Approach. Artificial Intelligence, 173, 1539-1558. http://dx.doi.org/10.1016/j.artint.2009.07.006

[35] Tang, Y. and Zheng, J. (2006) Linguistic Modelling Based on Semantic Similarity Relation amongst Linguistic Labels. Fuzzy Sets and Systems, 157, 1662-1673. http://dx.doi.org/10.1016/j.fss.2006.02.014 